\title{
Archéopages
}

Archéopages

Archéologie et société

$36 \mid 01 / 2013$

Exotismes

\section{Une séquence du Pléistocène moyen et supérieur en Charente}

\section{Nelly Connet}

\section{OpenEdition}

1 Journals

Édition électronique

URL : https://journals.openedition.org/archeopages/232

DOI : 10.4000/archeopages.232

ISSN : 2269-9872

Éditeur

INRAP - Institut national de recherches archéologiques préventives

Édition imprimée

Date de publication : 1 novembre 2013

Pagination : 74-75

ISSN : 1622-8545

\section{Référence électronique}

Nelly Connet, « Une séquence du Pléistocène moyen et supérieur en Charente », Archéopages [En

ligne], 36 | 01/2013, mis en ligne le 01 janvier 2015, consulté le 22 janvier 2022. URL : http:// journals.openedition.org/archeopages/232 ; DOI : https://doi.org/10.4000/archeopages.232 
de la vallée encaissée de La Péruse, petit affluent de la Charente, qui s'écoule à l'est. L'approche a été mécanisée au maximum, ce qui a permis la fouille extensive du site

\section{Une séquence du Pléistocène moyen et supérieur en Charente}

Nelly Connet

Inrap, UMR 7041 «Archéologie et Sciences de l'Antiquité », responsable d'opération

Site

La Grande Brousse

Londigny

Charente

Date

Avril-juin 2012

Surface fouillée

$3000 \mathrm{~m}^{2}$

Équipe

Géologie

Pascal Bertran, Inrap, UMR 5199

et l'exploration de l'ensemble de la rouge d'origine colluviale (horizon
Le site est localisé sur un plateau de calcaires jurassiques parsemé de petites dolines au niveau du Seuil du Poitou, dans la zone de contact entre bassin parisien et bassin aquitain. Il a été découvert lors d'un diagnostic réalisé par Frédéric Gerber (Inrap) en hiver 2011 sur le tracé de la future ligne à grande vitesse Sud-EuropeAtlantique (Gerber, 2011). Sylvain Soriano (CNRs) en avait alors réalisé l'étude des séries lithiques paléolithiques mises au jour.

L'emprise de la fouille est située sur le rebord du plateau, en surplomb couverture sédimentaire pléistocène contenant les vestiges,

exclusivement lithiques, qui atteint par endroits $3 \mathrm{~m}$ de puissance. La séquence sédimentaire observée comprend schématiquement au-dessus du substratum calcaire [ill. 1] : à la base, des argiles jaunes provenant de l'altération des calcaires, des limons argileux brun paléoargilique - IIBT -, environ 50 cm d'épaisseur), des limons brun jaune d'origine éolienne (environ $1 \mathrm{~m}$ d'épaisseur), des limons bruns (horizon argilique - BT - du sol holocène, $40 \mathrm{~cm}$ ). D'après l'étude micromorphologique, l'horizon IIBT est interprété comme un pédocomplexe qui représente le bilan de plusieurs interglaciaires du Pléistocène moyen.

Les limons supérieurs contenaient des vestiges archéologiques disséminés sur toute leur épaisseur, sans concentrations identifiables. Les datations par thermoluminescence sur silex chauffé, effectuées par Nick Debenham (Ouaternarv TL Survevs,
Nottingham), mettent en évidence au moins une phase d'occupation contemporaine du début glaciaire weichsélien (Stade isotopique marin $5 \mathrm{C}$-a). Deux autres pièces datées des SIM 7-8 (230 $\pm 54 \mathrm{ka})$ et 9 ( $307 \pm$ $37 \mathrm{ka}$ ) indiquent que du matériel correspondant à des occupations plus anciennes a été repris par colluvionnement et intégré au sein des limons supérieurs weichséliens. Malheureusement, il est difficile de discriminer le mobilier archéologique d'après sa position stratigraphique, ce qui rend assez illusoire toute étude technoculturelle.

Les ensembles archéologiques contenus dans ces limons se composent de petits bifaces, partiellement aménagés, à extrémité apicale aiguë [ill. 2], associés, entre autres, à un débitage de conception Levallois et à des outils sur éclats Celui du paléosol (horizon IIBT) est nettement plus intéressant. Il est présent sur 20 à $50 \mathrm{~cm}$ d'épaisseur et a pu être clairement identifié sur deux secteurs de la fouille, soit sur une surface totale d'environ $700 \mathrm{~m}^{2}$ correspondant à la partie basse de deux petites dépressions. Dans les autres secteurs du site, ce paléosol se confond avec le BT supérieur. La disposition des vestiges dans le sédiment est lâche, et aucune concentration n'a été constatée.

1

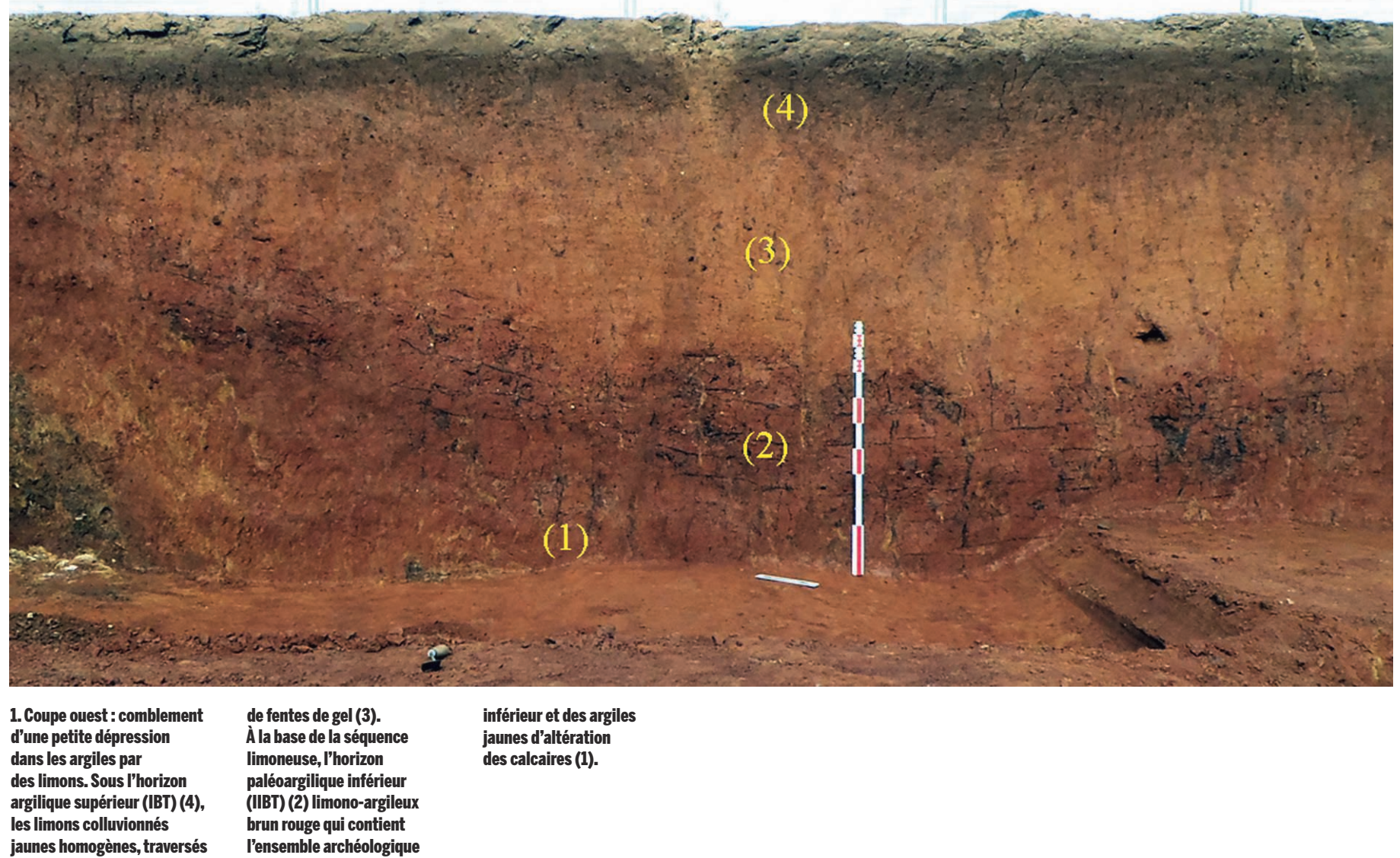



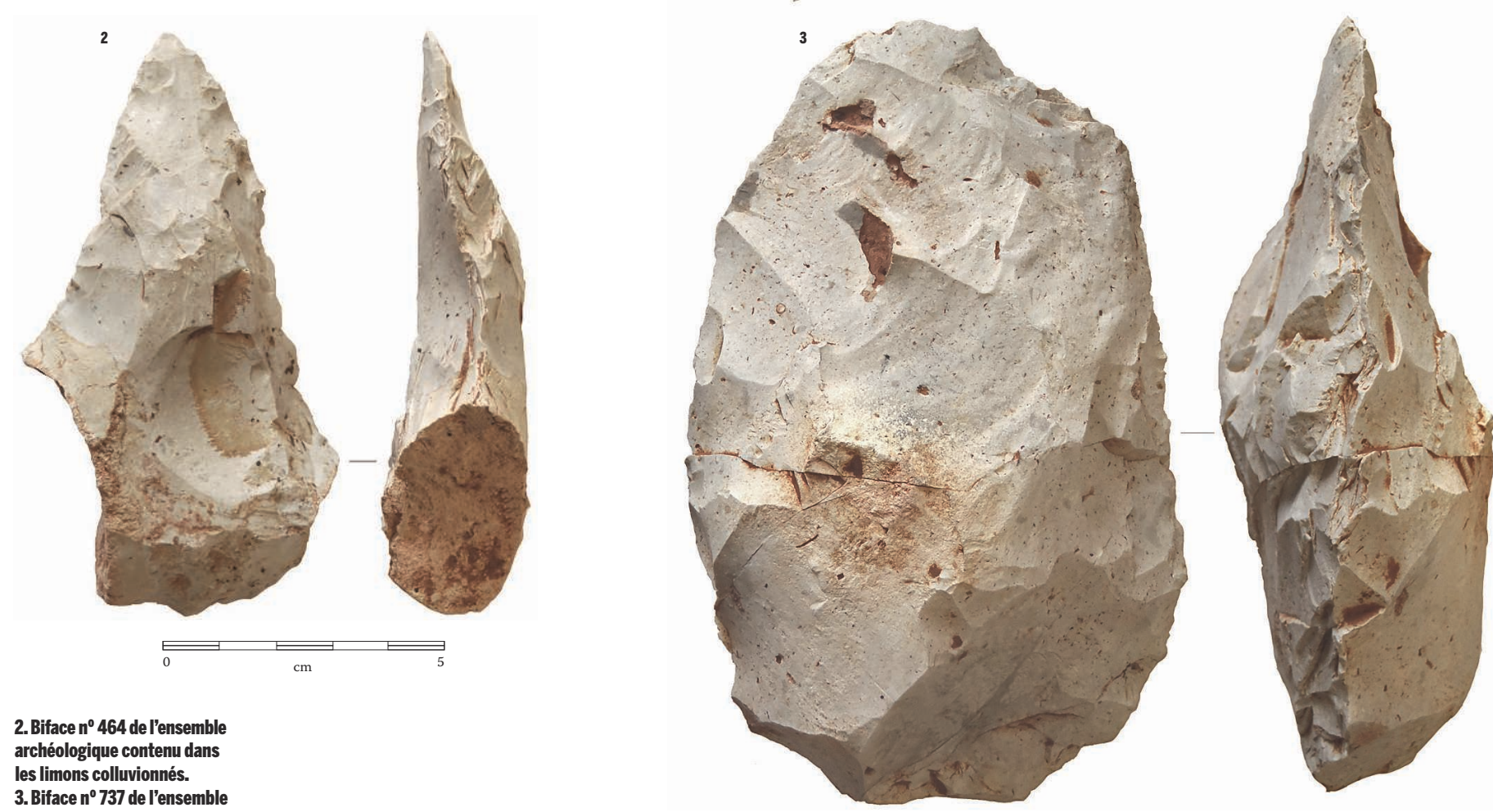

2. Biface $\mathrm{n}^{0} \mathbf{4 6 4}$ de l'ensemble

archéologique contenu dans

les limons colluvionnés.

3. Biface $n^{0} \mathbf{7 3 7}$ de l'ensemble

archéologique contenu dans

le paléosol (IIBT).

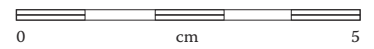

Aussi, aucun niveau archéologique n'est décelable. Les données géologiques suggèrent que l'horizon IIBT correspond sans doute au bilan pédosédimentaire de plusieurs interglaciaires du Pléistocène : le mobilier archéologique qu'il renferme peut donc relever de différentes séquences d'occupation. Les datations par thermoluminescence confirment l'ancienneté de la base de la séquence sédimentaire avec une date de $396 \pm 66 \mathrm{ka} \mathrm{BP}$ (QTLS LON26), soit SIM 10-12. Comme indiqué plus haut, certaines pièces provenant des limons supérieurs indiquent la présence d'autres occupations d'âge compris entre les $\mathrm{SIM}_{7}$ et 9.

Le paléosol renfermait 193 vestiges lithiques en excellent état de conservation, taillés dans un silex jurassique présent dans l'environnement immédiat du site. Ce petit ensemble se compose de nucléus, d'éclats bruts, d'outils sur éclats (denticules et éclats retouchés) et de pièces bifaciales. L'analyse technique des nucléus et des éclats montre un débitage simple, sans préparation des volumes, où une série d'enlèvements unidirectionnels est détachée depuis un plan de frappe naturel. Dans de rares cas, cet algorithme simple peut se répéter deux à trois fois sur le même nucléus de façon indépendante, généralement sur des surfaces différentes. Les éclats obtenus sont plutôt allongés, souvent épais, et présentent un talon lisse et ouvert. Quatre pièces bifaciales entières et trois fragments complètent le lot d'outils sur éclats. Il s'agit de pièces de grand module (170 $\mathrm{x} 85 \mathrm{~mm}$ pour la plus grande), qui pourraient être rangées dans les types amygdaloïdes, cordiformes à ovalaires de la classification de François Bordes (1961). Ils sont totalement ou partiellement aménagés, leur base est épaisse et seul le tiers supérieur de la pièce est réellement aminci [ill. 3], avec une partie apicale arrondie. Il s'agit de pièces bifaciales supports d'outils, c'est-à-dire d'outils aménagés sur des supports bifaciaux, et non de pièces bifaciales-outils, pour lesquelles la pièce bifaciale est conceptualisée dès l'ébauche pour n'être qu'un seul outil intégré à son support (Boeda, 1997).

Le système de production d'éclats, s'il n'est pas symptomatique d'un moment de l'histoire technique de l'Homme, est cohérent avec la présence de pièces bifaciales. Les outils sur éclats, tels denticules et encoches, constituent la panoplie de ceux qui sont le plus fréquemment associés aux pièces bifaciales durant le Paléolithique inférieur.
Dans le contexte général du Paléolithique inférieur en Europe de l'Ouest, les pièces bifaciales supports d'outils précèdent dans le temps les pièces bifaciales-outils et représentent les seules pièces bifaciales des sites du Paléolithique inférieur, avant le stade isotopique 9 (Nicoud, 2011). Le petit ensemble lithique de Londigny apparaît donc cohérent d'un point de vue technique et typologique et s'inscrit parfaitement dans la variabilité des gisements du Paléolithique inférieur connus en Europe entre les stades isotopiques 12 et 9 .

Dans cette partie médiane du Pléistocène moyen (plus de 150 ooo ans) et pour un territoire aussi vaste que l'ouest de l'Europe, nous décomptons tout au plus une cinquantaine de gisements en contexte stratigraphique, ce qui est peu. Ils sont principalement situés dans les bassins sédimentaires de la Tamise, de la Seine et de la Somme et dans les péninsules italienne et ibérique. Parce qu'il en est éloigné et qu'il s'inscrit au sein d'une vaste aire géographique, axe de passage naturel entre les plaines du Nord et le sud de l'Europe de l'Ouest, le site de Londigny constitue un jalon important dans la connaissance des civilisations de la partie médiane du Pléistocène moyen.

\section{Références bibliographiques}

BoËDA E., 1997 : Technogénèse des systèmes de production lithique au Paléolithique inférieur et moyen en Europe occidentale et au Proche-Orient, Mémoire d'habilitation à diriger les recherches, Université de Paris XNanterre, 2 vol., 173 p.

Bordes F., 1961 : Typologie du Paléolithique ancien et moyen, Bordeaux, Delmas (coll. Publications de l'Institut de préhistoire de l'université de Bordeaux, mémoire $\mathrm{n}^{\circ} 1$ ), 2 t., 85 p., $108 \mathrm{pl}$.

GERBER F. (DIR.), 2011: LGVSEA2 phase 32, PK 151,7 - PK 157,6, Tours, Angoulême, Rapport de diagnostic, Inrap, L'Isle d'Espagnac, $131 \mathrm{p}$.

Nicoud E., 2011 : Le phénomène acheuléen en Europe occidentale, Thèse de doctorat, Université de Aix-Marseille et Università degli Studi di Roma « La Sapienza », 483 p., 132 fig. 